\title{
A stem-loop structure directs oskar mRNA to microtubule minus ends
}

\author{
HELENA JAMBOR, ${ }^{1,3}$ SANDRA MUELLER, ${ }^{1}$ SIMON L. BULLOCK, ${ }^{2}$ and ANNE EPHRUSSI ${ }^{1,4}$ \\ ${ }^{1}$ European Molecular Biology Laboratory, 69117 Heidelberg, Germany \\ ${ }^{2}$ Cell Biology Division, MRC Laboratory of Molecular Biology, Cambridge CB2 0QH, United Kingdom
}

\begin{abstract}
mRNA transport coupled with translational control underlies the intracellular localization of many proteins in eukaryotic cells. This is exemplified in Drosophila, where oskar mRNA transport and translation at the posterior pole of the oocyte direct posterior patterning of the embryo. oskar localization is a multistep process. Within the oocyte, a spliced oskar localization element (SOLE) targets oskar mRNA for plus end-directed transport by kinesin-1 to the posterior pole. However, the signals mediating the initial minus end-directed, dynein-dependent transport of the mRNA from nurse cells into the oocyte have remained unknown. Here, we show that a 67-nt stem-loop in the oskar $3^{\prime}$ UTR promotes oskar mRNA delivery to the developing oocyte and that it shares functional features with the $f s(1) K 10$ oocyte localization signal. Thus, two independent cis-acting signals, the oocyte entry signal (OES) and the SOLE, mediate sequential dynein- and kinesin-dependent phases of oskar mRNA transport during oogenesis. The OES also promotes apical localization of injected RNAs in blastoderm stage embryos, another dynein-mediated process. Similarly, when ectopically expressed in polarized cells of the follicular epithelium or salivary glands, reporter RNAs bearing the oskar OES are apically enriched, demonstrating that this element promotes mRNA localization independently of cell type. Our work sheds new light on how oskar mRNA is trafficked during oogenesis and the RNA features that mediate minus end-directed transport.
\end{abstract}

Keywords: oskar; osk; Drosophila; oocyte; oogenesis; mRNA localization

\section{INTRODUCTION}

Early development of the transcriptionally silent Drosophila melanogaster embryo relies on maternal gene products deposited in the egg during oogenesis. The determinant patterning the posterior structures, the abdomen and germline, is Oskar protein, whose activity is spatially restricted to the posterior pole of the embryo (Lehmann and NussleinVolhard 1986). Embryos lacking Oskar fail to develop an abdomen and germline, whereas ectopic expression of Oskar at the anterior causes formation of germ cells and abdominal structures in place of the head (Lehmann and NussleinVolhard 1986; Ephrussi and Lehmann 1992; Smith et al. 1992). The localized pool of Oskar protein present in the syncytial embryo is generated during oogenesis by oskar mRNA localization and local translation at the posterior pole of the oocyte (Ephrussi et al. 1991; Kim-Ha et al. 1991, 1995; Markussen et al. 1995; Rongo et al. 1995).

\footnotetext{
${ }^{3}$ Present address: Max-Planck Institute of Molecular Cell Biology and Genetics, Pfotenhauerstrasse 108, 01307 Dresden, Germany

${ }^{4}$ Corresponding author

E-mail ephrussi@embl.de

Article published online ahead of print. Article and publication date are at http://www.rnajournal.org/cgi/doi/10.1261/rna.041566.113. Freely available online through the RNA Open Access option.
}

The Drosophila oocyte develops within an egg-chamber consisting of germline cells surrounded by a somatic follicular epithelium. Oogenesis has been divided into 14 developmental stages (King 1970). Through four rounds of mitosis with incomplete cytokinesis, a germline stem cell gives rise to one oocyte and 15 nurse cells. Throughout the course of oogenesis, the 16 germline cells remain interconnected by ring canals and share a microtubule cytoskeleton that allows the transport of molecules between cells in the cyst (for review, see Bastock and St Johnston 2008). During early oogenesis, oskar mRNA is transcribed in the nurse cells and subsequently enriched in the oocyte, where the mRNA is detected from stage 2 onward. At mid-oogenesis, oskar mRNA localizes briefly at the anterior margin of the oocyte (stages 7-8), then is enriched transiently in the center of the oocyte (stage 8), and finally accumulates at the posterior pole (stage 9) (Ephrussi et al. 1991; Kim-Ha et al. 1991). Oskar protein is detected from stage 9 onward, when oskar mRNA is within the posterior domain, and both molecules remain localized at the posterior pole until early embryogenesis (Kim-Ha et al. 1995; Markussen et al. 1995; Rongo et al. 1995). After

(C) 2014 Jambor et al. This article, published in RNA, is available under a Creative Commons License (Attribution-NonCommercial 3.0 Unported), as described at http://creativecommons.org/licenses/by-nc/3.0/. 
stage 9, oskar mRNA and Oskar protein continue to accumulate at the posterior pole, and this late phase of Oskar accumulation is important for proper patterning of the embryo (Snee et al. 2007; Sinsimer et al. 2011). The transport of oskar mRNA from the nurse cells into the oocyte is an essential first step toward production of Oskar posterior patterning activity; this process is also a prerequisite for female gamete production, as oocytes lacking oskar RNA arrest their development prematurely due to a noncoding function of this transcript (Jenny et al. 2006).

In eukaryotes, intracellular mRNA localization by active transport involves the cytoskeleton and associated motor proteins (for review, see Medioni et al. 2012). In Drosophila, the microtubule cytoskeleton has the major role in mRNA localization. Feeding flies with the microtubule depolymerizing drug colchicine results in accumulation of oskar mRNA in the nurse cells and its failure to enrich in the oocyte in stage 2-7 egg-chambers (Theurkauf et al. 1993; Pokrywka and Stephenson 1995). Treatment of mid-oogenesis egg-chambers with colchicine causes the mRNA to detach from the posterior pole (Theurkauf et al. 1993; Pokrywka and Stephenson 1995). Therefore, an intact microtubule cytoskeleton is required for oskar mRNA localization during early and midoogenesis. The accumulation of oskar mRNA during late oogenesis likely involves mechanistically distinct pathways and requires prelocalized oskar mRNA, Oskar Protein, and additional factors such as Rump and Lost (Glotzer et al. 1997; Jambor et al. 2011; Sinsimer et al. 2011).

During early oogenesis, the microtubule organizing center (MTOC) of the germline syncytium is restricted to the oocyte, and the microtubules that nucleate within this cell extend through the ring-canals into the neighboring nurse cells (Theurkauf et al. 1993); at mid-oogenesis, the microtubule network is reorganized such that the bulk of the microtubules nucleate at the anterior cortex and extend toward the posterior pole of the oocyte (Theurkauf et al. 1992; Parton et al. 2011); a distinct array of microtubules that emanates from a nuclear membrane-localized MTOC positions the oocyte nucleus at an antero-lateral position in the oocyte (Januschke et al. 2006; Zhao et al. 2012). Consistent with these observations, Nod- $\beta$ gal, a reporter of microtubule minus ends also enriches in the oocyte in young egg-chambers, whereas during mid-oogenesis, it is detected at the oocyte anterior, while Kinesin- $\beta$ gal, a reporter of microtubule plus ends, is enriched at the posterior pole (Clark et al. 1994, 1997). Thus, the distribution of oskar mRNA in the oocyte during the early stages and its anterior localization at stage 8 are consistent with microtubule minus end-directed transport.

In support of this notion, it has been shown that oskar mRNA accumulation in the early oocyte is dependent on the major minus end-directed motor cytoplasmic dynein: oskar mRNA colocalizes with the dynein complex proteins Dynein heavy chain (Dhc), Bicaudal D (BicD), and Egalitarian (Egl) in the oocyte of young egg-chambers, and disrup- tion of these factors affects oskar mRNA transport into the oocyte (Ephrussi et al. 1991; Suter and Steward 1991; Mach and Lehmann 1997; McGrail and Hays 1997; Swan et al. 1999; Clark et al. 2007). In contrast, mutations in Kinesin heavy chain (Khc), which encodes the motor component of kinesin-1, do not affect accumulation of oskar mRNA in the early oocyte. However, they specifically abrogate the posterior localization of the mRNA at stage 9 (Brendza et al. 2000; Cha et al. 2002; Parton et al. 2011), which is thought to result from the random, yet slightly biased movement of oskar mRNA to the posterior pole that presumably reflects the mild polarity bias of microtubules in that region (Zimyanin et al. 2008; Parton et al. 2011). Taken together, these data provide evidence that the microtubule-dependent transport of oskar mRNA from the nurse cells into the oocyte depends on dynein, whereas posterior localization following entry into the oocyte involves kinesin-1.

The cis-regulatory signals responsible for targeting localized transcripts (so-called zipcodes) (Singer 1993) are recognized by specific RNA binding proteins that coassemble with the RNA to form localization-competent RNPs associated with motor proteins. RNA zipcodes typically comprise features in primary sequence and/or secondary and tertiary structure (for review, see Martin and Ephrussi 2009; Bullock et al. 2010). In Drosophila, a few zipcodes active during oogenesis have been identified, such as the GLS of gurken mRNA (Van De Bor et al. 2005) and the BLE1 of bicoid mRNA (Macdonald et al. 1993; Macdonald and Kerr 1998). Another well-characterized localization signal is the 44-nt-long RNA stem-loop structure of $f_{s}(1) K 10$ that mediates oocyte enrichment and is sufficient to promote transport of unrelated RNAs into the young oocyte (Cohen et al. 2005; Bullock et al. 2010). Interestingly, the $f_{s}(1) K 10$ signal is active both in the oocyte and syncytial blastoderm embryos, where, upon injection, RNA comprising the signal is transported apically to the microtubule minus ends in a BicD- and Egldependent manner (Bullock and Ish-Horowicz 2001). Similarly, when expressed in the female germline, hairy mRNA, which localizes apically in the embryo, is targeted to the developing oocyte (Bullock and Ish-Horowicz 2001). Hence, RNA zipcodes for minus end-directed transport are recognized by motor complexes active both in egg-chambers and embryos.

oskar mRNA transport to the posterior pole requires the oskar 3' UTR and splicing of the first intron (Hachet and Ephrussi 2004). Splicing of this intron creates a short RNA stem-loop structure, the spliced oskar localization element (SOLE), in which complementary nucleotides of exons 1 and 2 are juxtaposed, forming a posterior targeting signal (Ghosh et al. 2012). Unspliced (and spliced) oskar mRNAs can also localize at the posterior pole by hitchhiking with actively transported oskar transcripts (Hachet and Ephrussi 2004). Hitchhiking involves direct RNA-RNA interaction between different oskar RNA molecules, via the terminal loop of a stem-loop structure (the dimerization domain) in the oskar 
3' UTR (Jambor et al. 2011). Using a genetic combination that results in flies entirely lacking endogenous oskar RNA, Hachet et al. demonstrated that the $3^{\prime}$ UTR alone can enrich in the oocyte and that the rest of the mRNA, including the SOLE, is dispensable for this initial transport step (Hachet and Ephrussi 2004). However, the exact nature of the RNA signal responsible for oskar mRNA enrichment in the oocyte has remained elusive. Identification and detailed characterization of the oskar oocyte entry signal would not only shed light on the molecular mechanisms underpinning axial patterning in Drosophila but would also contribute to understanding the general RNA features that underlie transport of mRNAs in eukaryotic cells. Indeed, a genome-wide in situ hybridization screen has revealed the remarkable prevalence of transcript localization in embryos, including a large number of transcripts enriched at the minus ends of microtubules (Lecuyer et al. 2007).

Here, we identify a signal in the oskar $3^{\prime}$ UTR, the oskar oocyte entry signal (OES), that is critical for mRNA transport into the oocyte. We provide evidence that secondary structural features and an AU-rich base-pair composition of this RNA segment are important for its mRNA transport function. Furthermore, we demonstrate that the OES also promotes apical mRNA enrichment in follicular epithelial cells, in blastoderm-stage embryos, and larval salivary glands. It was previously demonstrated that dynein mediates apical mRNA localization in follicular epithelial cells and in blastoderm embryos (Wilkie and Davis 2001; Horne-Badovinac and Bilder 2008; Li et al. 2008). Thus, our data indicate that, in addition to the SOLE, which mediates posterior localization within the oocyte, oskar mRNA harbors a dedicated signal, the OES, that promotes dynein-based mRNA accumulation in the oocyte.

\section{RESULTS AND DISCUSSION}

\section{A fragment of the oskar $3^{\prime}$ UTR mediates RNA enrichment in the oocyte}

Like oskar mRNA, several transcripts, including bicoid, gurken, and $f_{s}(1) K 10$, are enriched in the developing oocyte at stages $2-6$. The exact cis-regulatory signals mediating import of $f_{\mathcal{S}}(1) \mathrm{K} 10$ and bicoid mRNAs into the oocyte have been characterized (Macdonald et al. 1993; Serano and Cohen 1995; Macdonald and Kerr 1998). An early study suggested that a 2011). portion of the $3^{\prime}$ UTR was required for enrichment of oskar in the oocyte (Kim-Ha et al. 1993). While subsequent experiments revealed that the same region contains an RNA dimerization element that mediates reporter RNA hitchhiking with endogenous oskar transcripts to the posterior pole, this hitchhiking element did not appear to be involved in oocyte accumulation of oskar mRNA (Jambor et al. 2011). Hence, it was important to determine the exact signal mediating transport of oskar mRNA from the nurse cells into the oocyte.

It was previously shown that the $3^{\prime}$ UTR is sufficient for oskar mRNA accumulation in the oocyte (Jenny et al. 2006). To identify the minimal region mediating this early transport step, we generated transgenic flies expressing variants of the full-length oskar 3' UTR (Fig. 1A) fused to the open reading frame of Enhanced Green Fluorescent Protein (EGFP). Expression of this construct was under the control of a yeast UAS that can be activated by the transcription factor GAL4 expressed in a tissue-specific manner. The egfp sequences allow assessment of the localization of the
A

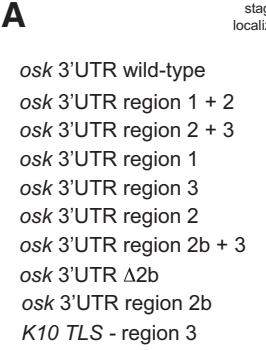

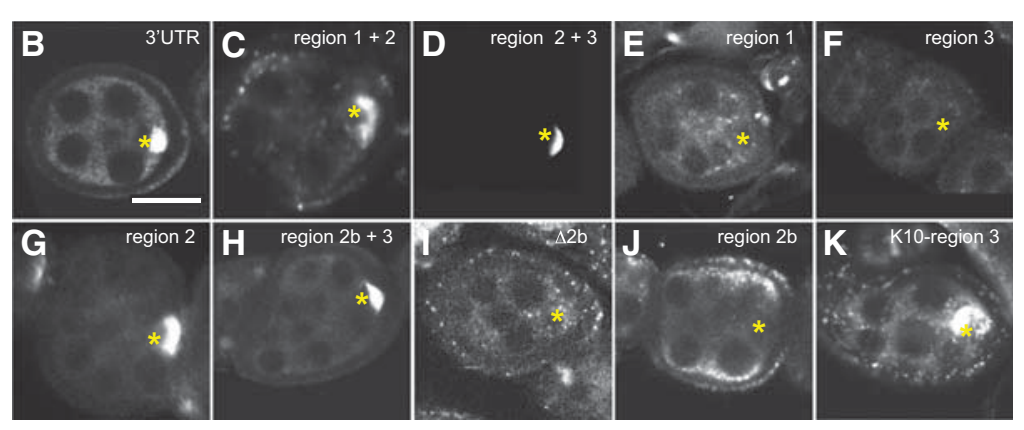

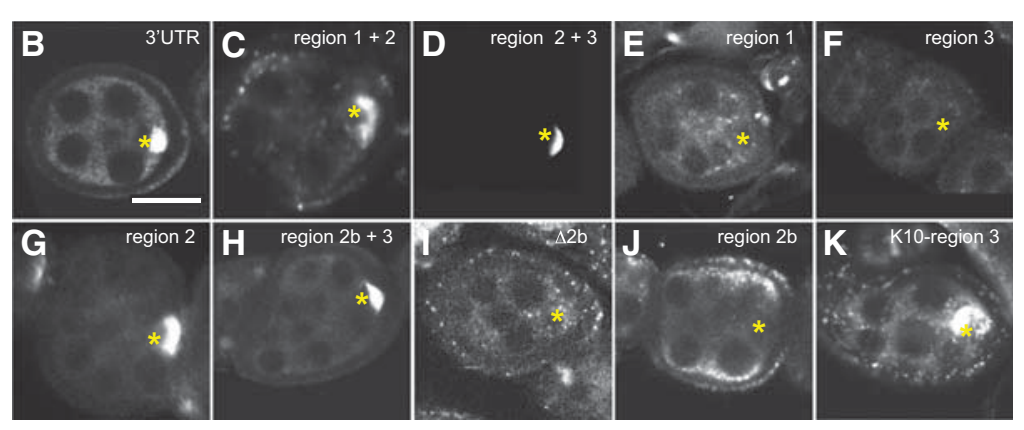

L

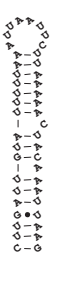

M

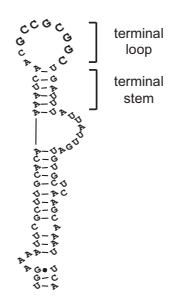

FIGURE 1. The central portion of oskar $3^{\prime}$ UTR is necessary and sufficient for oocyte transport. (A) Schematic representation of the reporter constructs analyzed in this panel and their ability to localize to the oocyte portion of stage 5-6 egg-chambers. Red rectangle: region $2 \mathrm{~b}$ encompassing the oocyte entry signal (OES), gray rectangle: TLS of $f_{s}(1) K 10$. Numbers indicate nucleotide positions within the oskar $3^{\prime}$ UTR. (B-K) Stage 5-6 egg-chambers from oskar RNA null flies (osk ${ }^{\text {A87/ }}$ $D f(3 R) p^{X T 103}$ ) expressing transgenic oskar $3^{\prime}$ UTR variants fused to the egfp open reading frame and under the control of a yeast UAS. Reporter mRNAs were expressed by $p$ Cog-Gal4 and nosGal4 drivers and detected by fluorescent in situ hybridization with an egfp-antisense probe. The asterisk indicates the position of the oocyte nucleus. Bar, $30 \mu \mathrm{m}$. The effects on localization shown were fully penetrant in all scored egg-chambers $(n \geq 20)$. Reporter mRNAs were fused to: the complete oskar $3^{\prime}$ UTR $(B)$; region $1+2(C)$; region $2+3(D)$; region $1(E)$; region $3(F)$; region $2(G)$; region $2 \mathrm{~b}+3$ of the oskar $3^{\prime}$ UTR $(H)$; the complete oskar $3^{\prime}$ UTR bearing a deletion of region $2 \mathrm{~b}(I)$; region $2 \mathrm{~b}$ of the oskar $3^{\prime} \mathrm{UTR}(J)$; region 3 fused at its $5^{\prime}$ end to the TLS of $f_{s}(1) K 10$ mRNA $(K) .(L, M)$ Experimentally validated secondary structures of the 44-nt-long TLS of $f_{S}(1)$ K10 mRNA (L) (Bullock et al. 2010) and of the 67-nt-long OES of oskar mRNA (M) (Jambor et al. 
reporter RNA variants by fluorescent in situ hybridization. oskar mRNA contains a dimerization domain that promotes intermolecular RNA:RNA interaction in vitro and posterior localization of transcripts lacking the SOLE by "hitchhiking" with endogenous oskar message in vivo (Jambor et al. 2011). Mutations in the dimerization domain that affect hitchhiking of RNAs to the posterior pole do not impair their entry into the oocyte (Jambor et al. 2011). Nevertheless, to unambiguously test the localization capacities of our reporters, we first expressed the chimeric oskar 3' UTR RNAs in the germline of oskar RNA null flies ( $o s k^{A 87} / D f(3 R) p^{X T 103}$ ) that express no endogenous oskar RNA (Hachet and Ephrussi 2004; Jenny et al. 2006; Jambor et al. 2011).

We analyzed the ability of the oskar 3' UTR RNA variants to accumulate in stage 5-6 oocytes, whose size readily allows one to distinguish localized from unlocalized mRNA distributions. As expected, full-length oskar 3' UTR (1028 nt) directed accumulation of egfp RNA in the oocytes of oskar RNA null flies in 100\% of egg chambers (Fig. 1B). Although the expression levels of the analyzed oskar 3' UTR variants were comparable as judged by qRT-PCR (data not shown), we observed striking differences in their pattern of accumulation in the oocyte. Deletions removing $499 \mathrm{nt}$ from the $5^{\prime}$ end (region 1) or $269 \mathrm{nt}$ from the $3^{\prime}$ end (region 3 ) of the 3' UTR did not impair RNA localization at stages 5-6 (region $1+2,2+3$ reporter RNAs) (Fig. 1C,D); reciprocally, neither region 1 nor region 3 , on its own, promoted reporter enrichment in the oocyte (Fig. 1E,F). Therefore region 1, which encompasses binding sites for the translational repressor Bruno (Kim-Ha et al. 1995), and region 3, which is required for oogenesis progression (Vazquez-Pianzola et al. 2011), are neither required nor sufficient for oocyte accumulation of oskar mRNA. In contrast, the central portion of the oskar $3^{\prime}$ UTR (region 2) alone provoked RNA enrichment in all oocytes examined (Fig. 1G). We therefore conclude that region 2 of the oskar $3^{\prime}$ UTR is both necessary and sufficient for enrichment of the RNA in the early oocyte.

We have previously demonstrated using thermodynamic folding algorithms, as well as by chemical and enzymatic probing, that region 2, which overlaps with the oskar RNA dimerization domain, folds into two stem-loop structures (Jambor et al. 2011). Deletion of the first stem-loop (region 2a) did not hamper the ability of the resulting region $2 b+3$ RNA to become enriched in oskar RNA null oocytes (Fig. $1 \mathrm{H})$. Since region 3 is not required for transport of oskar mRNA into the oocyte, we conclude that the localization activity is restricted to region $2 \mathrm{~b}$. Consistent with this notion, deletion of region $2 \mathrm{~b}$ from the full-length $3^{\prime}$ UTR abolished its oocyte enrichment in young egg-chambers (Fig. 1I). Interestingly, reporter RNA containing region $2 \mathrm{~b}$ alone could not be detected in the oocyte of oskar RNA null flies (Fig. 1J). Thus, additional flanking sequences may be required for proper folding of region $2 \mathrm{~b}$. Altogether, our data show that region $2 \mathrm{~b}$ (nt 630-759) mediates the enrichment of oskar RNA in the oocyte during early oogenesis.

\section{A stem-loop is required for transport of oskar mRNA into the oocyte}

Both oskar and $f_{\mathcal{S}}(1)$ K10 mRNAs localize to the oocyte in young egg-chambers (stages 2-7). We therefore asked whether the oocyte targeting signals of the two RNAs are structurally and functionally related. To test this, we generated a reporter consisting of the EGFP open reading frame, followed by the transport/localization signal (TLS) of the $f_{\mathcal{s}}(1) \mathrm{K} 10$ mRNA (Serano and Cohen 1995) and the nonlocalizing region 3 of the oskar $3^{\prime}$ UTR. When expressed in the germline of oskar RNA null flies, this chimeric RNA reporter fully recapitulated the early localization of oskar mRNA in the oocyte (Fig. $1 \mathrm{~K}$ ), demonstrating that the oocyte localization signals of oskar mRNA and $f_{s}(1) K 10$ are interchangeable as previously proposed (Serano and Cohen 1995).

The $f_{s}(1) K 10$ TLS forms a 44-nt stem-loop (Fig. 1L; Serano and Cohen 1995; Bullock et al. 2010), whereas oskar region 2b was shown to form a stem-loop $114 \mathrm{nt}$ long (Fig. 1M; Jambor et al. 2011). To test which portion of the oskar region $2 \mathrm{~b}$ stemloop is required for oocyte transport, we created versions of the region 2 egfp reporter in which either the proximal (lower) or the distal (upper) stem of region 2b was deleted (Fig. 2A). While reporter mRNA lacking the proximal stem (region 2 $\Delta 2 b-p)$ enriched in the developing oocyte similar to the control oskar region 2 mRNA (Fig. 2D,E), deletion of the distal stem and terminal loop entirely abolished oocyte entry (region $2 \Delta 2 b-d$ ) (Fig. 2F). These data demonstrate a critical role of the distal $67 \mathrm{nt}$ of region $2 \mathrm{~b}$ for oskar mRNA oocyte entry. However, the palindromic terminal loop that constitutes the oskar RNA dimerization domain is dispensable for oocyte enrichment, as point mutations within this region (Jambor et al. 2011) or deletion of the entire loop (Fig. 2G) caused no RNA transport defect.

The 67-nt distal portion of region $2 \mathrm{~b}$ is composed of a highly conserved stem interrupted by one large and several small bulges (Figs. 1M, 2B). Apart from one compensatory $A \rightarrow G$ substitution that should still allow base-pairing at this position, the terminal part of the stem, six bp long, is entirely conserved among Drosophilids (Fig. 2B). In the case of $f_{\mathcal{s}}(1) \mathrm{K} 10 \mathrm{mRNA}$, the most terminal stem-which is AU rich - was also shown to be the portion critical for transport during oogenesis (Serano and Cohen 1995). As there is no obvious common motif in the primary sequence of oskar region $2 \mathrm{~b}$ and the $f_{\mathcal{s}}(1)$ K10 TLS (Fig. 1L,M), we tested the importance of the secondary structure of the terminal stem of region $2 \mathrm{~b}$ for mRNA transport. We introduced point mutations ( $5^{\prime} m u t$ and $\left.3^{\prime} m u t\right)$ designed to disrupt base-pairing while maintaining the AU-composition of this region (Fig. 2C). Both $5^{\prime} m u t$ and $3^{\prime} m u t$ mRNAs had a reduced ability to localize in oocytes: $5^{\prime}$ mut mRNA was enriched in only $24 \%$ and $3^{\prime} m u t$ in $3 \%$ of oocytes at stage 6 (Fig. $2 \mathrm{H}$,I). The enrichment of $5^{\prime} m u t$ in a proportion of oocytes might be due to the formation of a new AU-rich stem (predicted by $m f o l d$ ) in $5^{\prime} m u t$ involving several nucleotides of the 


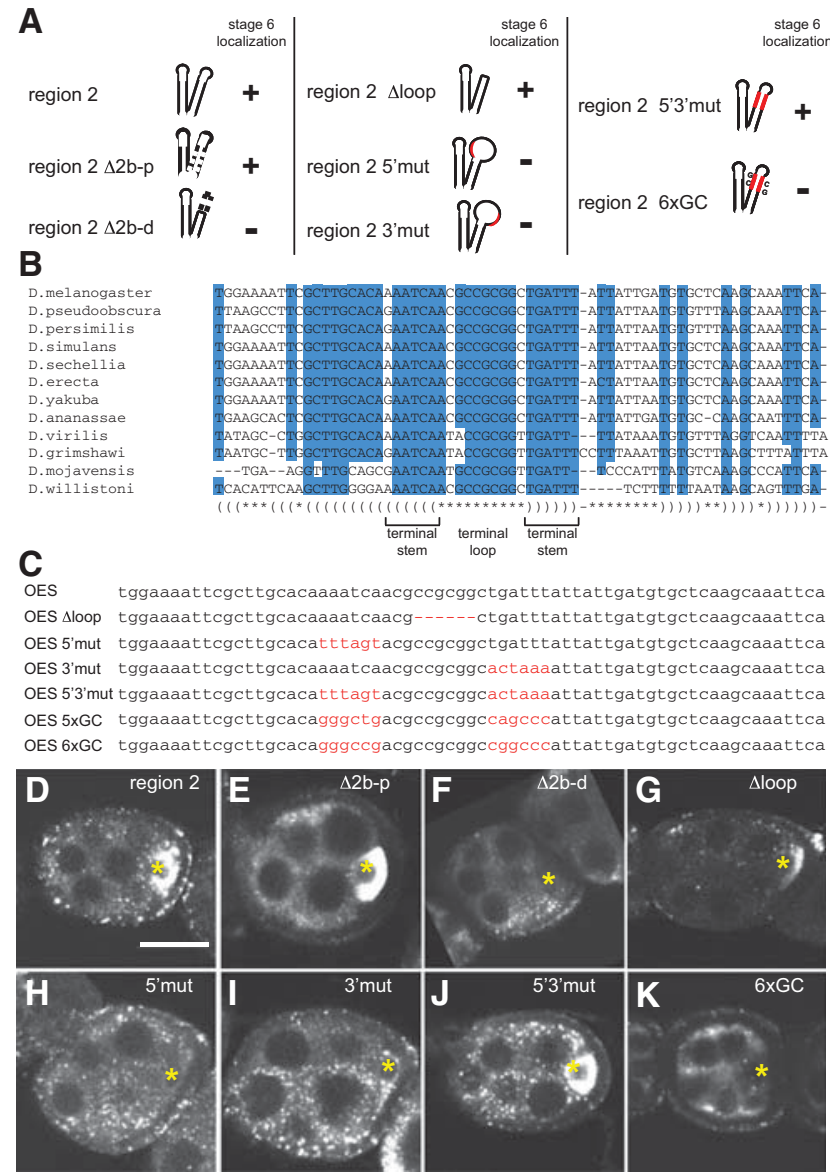

FIGURE 2. Region $2 \mathrm{~b}$ forms a stem-loop important for oocyte entry of oskar mRNA. (A) Schematic of the secondary structure of the reporter mRNAs analyzed in this panel and their localization capacity during early oogenesis. Red indicates stretches with point mutations. (B) Alignment of the OES region in 12 Drosophila species with sequenced genomes. Highlighted in blue are nucleotides with $>90 \%$ similarity. The experimentally validated secondary structure of the $D$. melanogaster OES is depicted below: A parenthesis indicates a base-paired nucleotide; an asterisk indicates a single-stranded nucleotide. Note that the terminal stem is almost entirely conserved among all species. (C) Primary sequence of the wild-type oocyte entry signal and the mutations analyzed in this panel. $(D-K)$ Stage 5-6 egg-chambers from wild-type flies expressing transgenic oskar 3' UTR-region 2 reporter mRNAs fused to the EGFP open reading frame under the control of mat-a4-tub-Gal4. Reporter RNAs are detected by fluorescent in situ hybridization using an egfp-antisense probe. A star indicates the position of the oocyte nucleus. Bar, $30 \mu \mathrm{m}$. Reporter mRNAs were fused to: region 2 of the oskar $3^{\prime}$ UTR $(D)$; region 2 with deletion of proximal stem of region $2 \mathrm{~b}: \Delta 2 \mathrm{~b}-\mathrm{p}$ $(E)$; region 2 with a deleted distal stem of region $2 \mathrm{~b}: \Delta 2 \mathrm{~b}-\mathrm{d}(F)$; region 2 with a deletion of the terminal loop $(G)$; region 2 with a mutated $5^{\prime}$ stem $(H)$; region 2 with a mutated $3^{\prime}$ stem $(I)$; region 2 with mutations in both the $5^{\prime}$ and $3^{\prime}$ stem such that complementarity is restored $(J)$; region 2 in which the quality of the terminal stem is changed from AU-rich to GC-rich $(K)$.

terminal stem (Supplemental Fig. 1). Restoration of the secondary structure by introduction of compensatory mutations that should restore base-pairing in the terminal stem $\left(5^{\prime} 3^{\prime}\right.$ mut) (Fig. 2J; Supplemental Fig. 1) improved RNA localization in stage 6 egg oocytes (58\% of oocytes showed oskar enrichment). These data suggest that the secondary structure, but not the nucleotide sequence, of oskar region $2 \mathrm{~b}$ is critical for RNA enrichment in the oocyte.

Next, we analyzed the importance of base-pair composition in the terminal stem for RNA localization. Of the six base pairs that form the terminal stem, five are $\mathrm{AU}$ and one is a GC base pair. Mutation of the AU-richness of the stem into GC-richness impaired oocyte accumulation of the resulting $5 x G C$ reporter RNA (enrichment in $41 \%$ of oocytes) (data not shown), and replacement of all AU base pairs by GC entirely abrogated RNA enrichment in all oocytes (region $26 \mathrm{xGC}$ ) (Fig. 2A,C,K). Our data suggest that not only the secondary structure but also AU-richness of the terminal stem impacts on the early transport of oskar mRNA.

Taken together, our results show that a 67-nt stem-loop structure in the $3^{\prime}$ UTR that we term the oocyte entry signal drives enrichment of oskar mRNA in the oocyte. The oskar OES and the $f_{s}(1) K 10$ TLS stem-loop structures appear to be functionally equivalent. In addition, the capacity of both RNA elements to mediate oocyte import of RNAs requires integrity of their terminal AU-rich stems. It is intriguing that two different features of the same stem-loop structure regulate two distinct events in oskar mRNA transport: the OES terminal stem is required for oocyte enrichment (this study), and the terminal loop for oskar RNAdependent hitchhiking to the posterior pole (Jambor et al. 2011).

\section{Dynein-mediated apical transport in syncytial embryos requires the oskar entry signal}

Multiple lines of evidence suggest that dynein transports oskar mRNA from the nurse cells to the early oocyte (see Introduction). Thus, the OES appears to be a zipcode for transport by this motor. To further test this notion, we injected and observed the localization of fluorescent, in vitro-synthesized RNAs in living Drosophila blastoderm-stage embryos. In this system, dynein-based transport of injected mRNAs to the apical cytoplasm (where minus ends of microtubules are enriched) has been extensively characterized (Bullock and Ish-Horowicz 2001; Wilkie and Davis 2001). In control experiments, we demonstrated that the $f_{\mathcal{S}}(1) \mathrm{K} 10$ mRNA was enriched apically following injection and that this was dependent on an intact TLS sequence (Fig. 3A,B). This observation is consistent with the results of earlier studies (Bullock and Ish-Horowicz 2001; Bullock et al. 2010). Also consistent with previous findings, the full-length oskar mRNA was enriched apically following injection (Fig. 3C), albeit with a less polarized distribution than $f_{s}$ (1)K10 (Bullock and Ish-Horowicz 2001). While the oskar region $2+3$ RNA displayed enrichment in the apical cytoplasm (Fig. 3D), this was not the case for region 3 alone (Fig. 3H). Furthermore, oskar reporter RNAs, in which the complete stem-loop 2b (Fig. 3E) or the OES (Fig. 3F) was deleted, were strongly impaired for apical localization. 


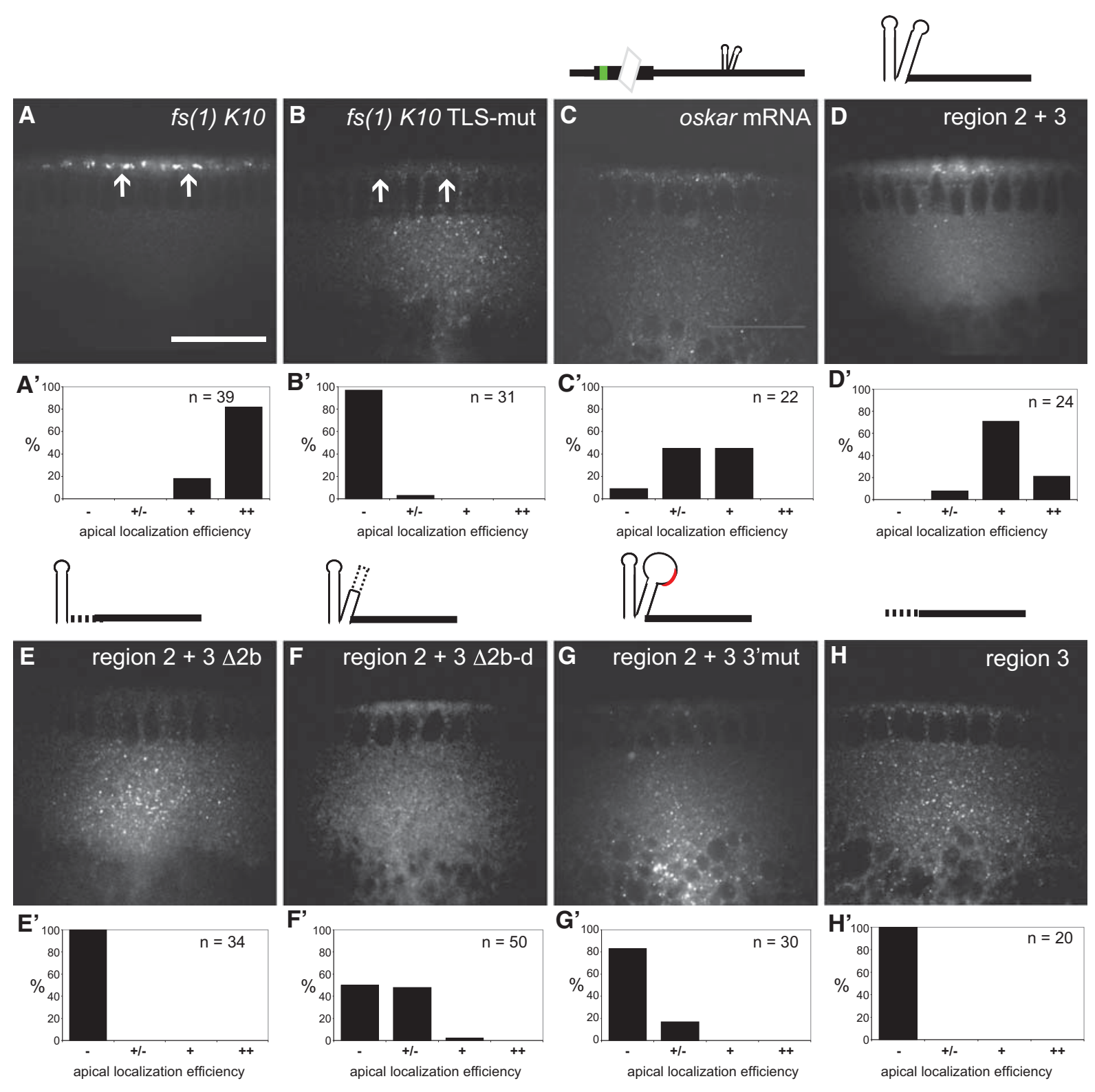

FIGURE 3. Region $2 \mathrm{~b}$ directs apical localization of transcripts in blastoderm embryos. $(A-H)$ RNA transcripts were injected into blastoderm embyos and fixed 8-10 min later (i.e., $8 \mathrm{~min}$ after injection of the last embryo). Images are oriented with apical to the top and basal to the bottom. Bar, $30 \mu \mathrm{m}$. The arrows in $A$ and $B$ indicate the apical cytoplasm of the blastoderm embryo. Schematic representations of the reporter RNAs are shown above the images. Thin black line: UTR RNAs, thick black line: ORF, dashed line: deletions, stem-loop: oocyte entry signal (OES) region, green rectangle: spliced oskar localization element (SOLE). $\left(A^{\prime}-H^{\prime}\right)$ Categorization of RNA enrichment in the apical cytoplasm. Percentage of embryos showing different efficiencies of transport is shown: $(++)$ strong, $(+)$ weak, $(+/-)$ very weak, $(-)$ no enrichment. (n) Number of embryos scored for each RNA. The following reporter mRNAs were injected: $f_{s}(1) K 10$ RNA, containing the entire $3^{\prime}$ UTR and flanking $3^{\prime}$ sequences $(A-$ $\left.A^{\prime}\right)$; a mutated version of the $f_{s}(1) K 10$ RNA, containing a randomized TLS sequence (Bullock et al. 2010) $\left(B-B^{\prime}\right)$; full-length oskar mRNA $\left(C-C^{\prime}\right)$; region $2+3$ of oskar $3^{\prime}$ UTR $\left(D-D^{\prime}\right)$; region $2+3$ of oskar $3^{\prime}$ UTR bearing a deletion of the region $2 \mathrm{~b}$ stem $\left(E-E^{\prime}\right)$; region $2+3$ of oskar $3^{\prime}$ UTR with a deletion of the distal stem $2 \mathrm{~b}\left(F-F^{\prime}\right)$; region $2+3$ of oskar $3^{\prime}$ UTR with a mutated $3^{\prime}$ portion of the terminal stem $\left(G-G^{\prime}\right)$; region 3 of oskar $3^{\prime} \operatorname{UTR}\left(H-H^{\prime}\right)$.

The $3^{\prime}$ stem mutation of the OES ( $\left.3^{\prime} m u t\right)$ also disrupted apical enrichment of RNA following injection (Fig. 3G). While these data show that the OES is necessary for apical enrichment in Drosophila embryos, a transcript consisting of region 2 alone failed to accumulate apically (data not shown). It is possible that, for stable folding, region 2 re- quires the context of a longer RNA sequence. Indeed, the localization activity of region 2 during oogenesis was assayed in a context where it was fused downstream from the EGFP open reading frame. Previous experiments have shown that short RNAs harboring apical zipcodes are transported inefficiently in the embryo (Dienstbier et al. 2009). 
Alternatively, there may be qualitative or quantitative differences in the localization machineries active during oogenesis and embryogenesis. Consistent with this notion, stem-loop IV/V, promoting oocyte localization of bicoid mRNA, was essential but not sufficient for robust apical transport in embryos (Snee et al. 2005).

Taken together, these data show that the oskar oocyte import signal also promotes the targeting of RNA apically in embryos, which is a dynein-dependent process. It is interesting to note that, although it contains an intact OES, the complete oskar mRNA shows less strong apical enrichment in Drosophila embryos than region $2+3$ alone (Fig. $3 \mathrm{C}, \mathrm{D})$. This might be due to a negative influence of other portions of oskar mRNA on the ability of the OES and associated proteins to form a functional RNA:motor complex in the embryo. Alternatively, it might reflect competition between the OES and other RNA signals, such as the posterior-targeting SOLE (Ghosh et al. 2012). This latter element is located within the open reading frame and drives kinesin-based transport of oskar mRNA to the oocyte posterior pole.

\section{Anterior localization at stage 8 also involves the oocyte entry signal}

After enriching in the developing oocyte at stages $2-7$ of oogenesis, and prior to its posterior localization, oskar mRNA is briefly detected at the anterior cortex (Ephrussi et al. 1991; $\mathrm{Kim}-\mathrm{Ha}$ et al. 1991). The anterior localization of $f_{\mathcal{S}}(1) \mathrm{K} 10$ transcripts requires the same RNA motif that is used for their transport into the oocyte (Serano and Cohen 1995). We therefore assessed whether the OES signal also drives oskar mRNA to the anterior cortex. As oskar RNA null flies arrest oogenesis at stage 7 (Jenny et al. 2006), we expressed the reporter RNAs in wild-type $\left(w^{1118}\right)$ flies and probed their localization at stage 8 of oogenesis by in situ hybridization to egfp sequences in the reporter. The presence of endogenous oskar mRNA would not influence these experiments, as indirect localization through hitchhiking mediates the posterior localization of intronless reporter RNAs at stage 9 but not their anterior enrichment (see Fig. $3 \mathrm{H}, \mathrm{M}$ in Jambor et al. 2011).

Reporter RNAs bearing either the full-length oskar 3' UTR RNA or fragments containing the intact oskar oocyte entry signal (region $1+2$, region $2+3$, region 2 ), each of which was enriched in young oocytes (Fig. 1B,C,D,G), accumulated at the anterior cortex at stage 8 (Fig. 4A-D) like oskar mRNA (Ephrussi et al. 1991; Kim-Ha et al. 1991). Furthermore, disruption of the OES (region $23^{\prime} m u t$ ) abolished RNA localization at the anterior cortex (Fig. 4E). In line with its effect on oocyte entry, restoring the terminal stem $\left(5^{\prime} 3^{\prime} m u t\right)$ reestablished anterior RNA localization at stage 8 (Fig. 4F). We therefore conclude that the same RNA features mediate oskar transport into the oocyte and subsequent localization at its anterior cortex.

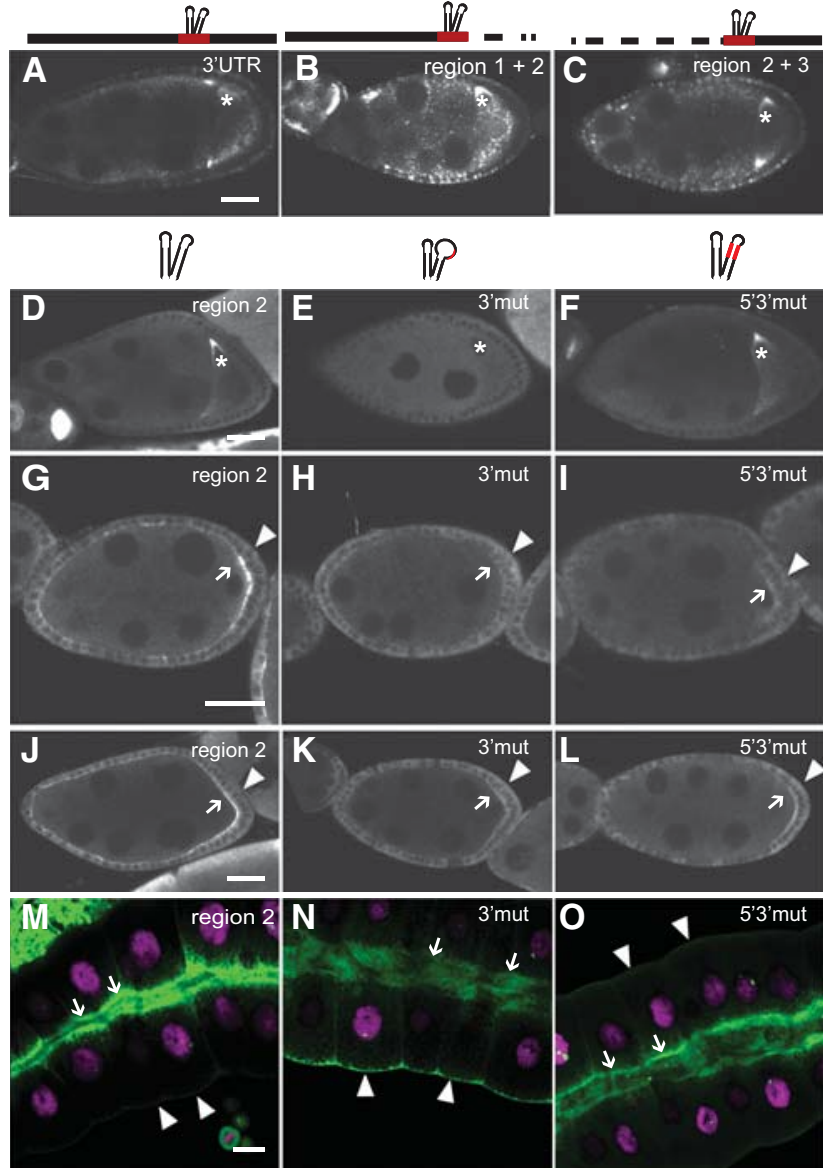

FIGURE 4. The oocyte entry signal mediates anterior localization in stage 8 oocytes and apical localization in polarized epithelia. $(A-O)$ Transgenic UAS-oskar $3^{\prime}$ UTR reporters fused to the egfp open reading frame were expressed under the control of the mat-a4-tub-Gal4 in the germline $(A-F)$, GR1-Gal4 in follicular epithelial cells $(G-L)$, or forkhead-Gal4 in salivary glands $(M-O)$. Reporter RNAs were detected by fluorescent in situ hybridization using an egfp-antisense probe (RNA shown in green in $M-O)$. For orientation, the salivary glands $(M-O)$ were counter-stained with DAPI to reveal DNA (shown in magenta). A star indicates the position of the oocyte nucleus $(A-F)$. Arrows indicate the apical membrane and arrowheads the basal membrane of salivary glands and follicle cells $(G-O)$. Bar, $30 \mu \mathrm{m}$. Above each image is a schematic representation of the reporter RNA analyzed. Reporter mRNAs were fused to: the oskar $3^{\prime}$ UTR $(A)$; region $1+2(B)$; region $2+3(C)$; wild-type region $2(D, G, J, M)$; region $23^{\prime}$ mut $(E, H, K, N)$; region $25^{\prime} 3^{\prime}$ mut $(F, I, L, O)$.

\section{The oskar entry signal directs apical mRNA localization in cells of the follicular epithelium and the salivary gland}

The above results suggested that the OES is a zipcode for microtubule minus end-directed transport in the germline and in early embryos. To test if the OES has a more general role in mediating asymmetric RNA localization, we expressed our egfp RNA reporters ectopically in other highly polarized cells. We first forced reporter expression in the somatic follicular epithelium of the egg-chamber using the GR1-GAL4 driver and examined RNA localization in these cells at stages $6-8$ 
of oogenesis. We found that, as early as stage 5, the egfp reporter RNA fused to region 2 showed an apical accumulation in all follicle cells (Fig. 4G), where it was detected until stage 9 (Fig. 4J). Mutations disrupting the terminal stem ( $\left.3^{\prime} m u t\right)$ strongly reduced the apical enrichment of the reporter RNAs in follicle cells at both stage 5 and stage 8 (Fig. $4 \mathrm{H}$, $\mathrm{K})$. When expressing region 2 RNA bearing the compensatory $5^{\prime} 3^{\prime}$ mutation $\left(5^{\prime} 3^{\prime} m u t\right)$, a weak enrichment of the apical reporter mRNA was detected (Fig. 4I,L).

A weak apical enrichment in follicle cells was previously documented for a reporter mRNA fused to the entire oskar 3' UTR expressed under heat-shock promoter control (Karlin-Mcginness et al. 1996). Our data show that the terminal stem of region $2 \mathrm{~b}$ is required for apical localization of oskar mRNA in follicle cells. Several mRNAs have been shown to localize to the apical membrane of the follicle cells in a dynein-dependent mode, such as std (Horne-Badovinac and Bilder 2008) and upd mRNAs (Van De Bor et al. 2011). However, the features of their respective transport signals and their similarity to the OES are, so far, unclear.

We also assayed the activity of the OES in the highly polarized cells of the larval salivary gland. During the growth of the larva, the salivary gland cells secrete essential glue proteins from their apical side into the central lumen of the gland. The orientation of the microtubules in these cells has been inferred from the localization of motor proteins that move toward the minus or plus ends (nod-lacZ and kinesin-lacZ, respectively) (Myat and Andrew 2002). It was concluded that the minus ends of microtubules are enriched near the apical surface (proximal to the gland lumen), with plus ends extending toward the basal side of the cell (Myat and Andrew 2002). When expressing the region 2 reporter in salivary glands, we observed a striking accumulation of the RNA at the apical membrane facing the gland lumen (Fig. 4M). In contrast, the $3^{\prime} m u t$ reporter RNA displayed a greatly reduced apical accumulation, with the RNA detected at both the apical and the basal membrane of the cell (Fig. $4 \mathrm{~N}$ ). The doubly mutant $5^{\prime} 3^{\prime}$ mut reporter RNA, which bears compensatory mutations in the OES that partially restore its function in the egg-chamber, was transported predominantly to the apical membrane in the salivary gland cells (Fig. 4O). In salivary glands of flies expressing the $f k h$-Gal4 driver alone, no egfp in situ signal was observed, indicating specificity of the detection method (data not shown). Our data show that the cellular machinery required to recognize and interpret the oskar OES is present and functional both in follicular epithelial cells and salivary gland cells.

We find that the 67-nt-long OES promotes transport of oskar and heterologous RNAs from the nurse cells into the oocyte and that it also drives the targeting of RNAs apically in embryos, follicle cells, and salivary glands. These observations suggest that this signal represents a cell-type-independent zipcode for microtubule minus end-directed transport. It is noteworthy that the OES shares some similarity in secondary structure with the oocyte transport and an- terior localization signal of $f_{\mathcal{s}}(1) \mathrm{K} 10$ (Fig. 1L,M), as well as with the stem-loop elements that drive minus end-directed transport of gurken during oogenesis and hairy and wingless RNAs during embryogenesis (Serano and Cohen 1995; Bullock et al. 2003, 2010; Van De Bor et al. 2005; dos Santos et al. 2008). In the future, it will be interesting to decipher the critical features defining this zipcode. Recently, a global analysis identified 134 transcripts among 3370 assayed that show apical localization in D. melanogaster embryos (Lecuyer et al. 2007) and which, therefore, may harbor a signal with activity similar to that of the oskar OES and $f_{s}(1)$ K10 TLS. In light of such pervasive mRNA localization, it will be interesting to determine whether colocalized mRNAs share a common zipcode and, furthermore, how these zipcodes are interpreted in other cell types and related species.

One candidate to directly bind the OES is the Egl protein. Egl is a noncanonical RNA binding protein that, together with its binding partner BicD, links minus end-directed RNA zipcodes of several RNAs that localize in oocytes and embryos using dynein (Dienstbier et al. 2009). Indeed, it has been shown that Egl is recruited to apically localized oskar RNA in the embryo (Bullock and Ish-Horowicz 2001).

Our demonstration that, in addition to the SOLE element for posterior localization, oskar mRNA contains a signal for oocyte entry and anterior localization reveals that a single mRNA can simultaneously harbor opposing zipcodes. The fact that these signals target the RNA to distinct locations indicates that these conflicting RNA elements must be tightly regulated over the course of oogenesis. Specifically, the OES, which drives dynein-based transport of oskar mRNA into the oocyte during early oogenesis, must somehow be deactivated or overruled at the transition from stage 8 to 9 to permit the kinesin-1-based posterior localization of the mRNA. By precisely deciphering the zipcodes that control transport of mRNAs by different molecular motors and how they interact within a single RNA, it should ultimately be possible to predict the intracellular destination of mRNAs.

Our work further highlights the fact that several cis-regulatory signals can occur within a single RNA secondary structure. We have identified the terminal stem of region $2 \mathrm{~b}$ as being critical for oocyte entry, while the terminal loop of the same structure was previously shown to promote mRNA hitchhiking (Jambor et al. 2011). Such a dense organization of regulatory signals also emphasizes the importance of precisely mapping functional elements in mRNAs.

The posterior activity of Oskar protein is essential for abdomen and germ cell formation in the embryo, but the extent to which the different steps of oskar mRNA localization contribute to embryonic patterning remains an open question. Oocyte accumulation of oskar mRNA early is essential for oogenesis progression (Jenny et al. 2006) and is, therefore, a prerequisite for posterior localization of the mRNA during mid-oogenesis. The mid-oogenesis localization of oskar mRNA is required to generate an initial pool of posterior Oskar protein, which is critical for anchoring the mRNA 
at the posterior pole (Markussen et al. 1995; Rongo et al. 1995). In addition, it has been shown that, during the late stages of oogenesis (10B onward), oskar mRNA undergoes another phase of accumulation and translation that contributes importantly to embryonic patterning (Snee et al. 2007; Sinsimer et al. 2011). This accumulation involves movement of oskar mRNA throughout the oocyte by cytoplasmic streaming and anchoring via a mechanism involving Rump, Lost, and Oskar protein itself (Glotzer et al. 1997; Sinsimer et al. 2011) and coincides with the dumping of nurse cell cytoplasm-including any residual oskar mRNA, presumably independent of any OES-mediated transportinto the oocyte. Our work describing the zipcode for oocyte entry should enable further dissection of different phases of oskar mRNA localization and determination of their respective contributions to embryonic patterning.

\section{MATERIALS AND METHODS}

oskar 3' UTR: A StyI-MaeIII fragment of the plasmid pBS-oskar 3' UTR was subcloned into the StuI site of the pUT7 vector, a pUC18 derivative containing a T7 RNA polymerase. oskar 3' UTR variants were PCR-amplified from pUT7 templates with primers attaching BamHI restriction sites, and TA cloned. BamHI fragments were then subcloned into pUASpGW $\triangle \mathrm{K} 10$ to generate the respective egfp RNAs. To generate $\mathrm{pUASpGW} \Delta \mathrm{K} 10$, the K10 terminator was removed from pUASp (Rorth 1998) by XbaI and PstI digestion, followed by blunting and religation of pUASp. The egfp open reading frame (ORF) was amplified from pCRII-TOPO-EGFP with primers attaching KpnI and NotI sites, digested, and cloned to KpnI-NotI-digested pUASp $\Delta \mathrm{K} 10$. The K10-region 3 construct was generated by blunt cloning a double-stranded (ds) DNA fragment containing the $f s(1) K L 10$ TLS to the $5^{\prime}$ end of the oskar $3^{\prime}$ UTR region 3 . The ds DNA encompassing the $f s(1) K 10$ TLS was generated by primer alignment. oskar 3' UTR region 2 mutants were generated by PCR mutagenesis (Quick Change PCR kit, Qiagen). The following oskar fragments were analyzed (deletions: [...], mutations: capital letters):

oskar region 1

(gatcgagatctcgatcccgcgaaattaatacgactcactataggcaaggaaccctatctgttctgg aacaaaatcggtcggcagcgcgtactgcaagttattgaaacgagtctggagtattaagttggg ttcttaatcaagatacatatatgcaaattttgactgggctggcaccggaaccgacaaaataag aactttttgatgattttacgatttacgctgatggatcgctgcttttacggaattcgcttagttttaat atgttttatatgtagtatgttctctgtctttgtttatttatatgttcgtgcacttgtcctagtccattattgtat attattgtgtgttttgtgttctatgttagatttaaacttctcaatttttcgctgtctgtgatttgttttg ccaatgccattgattttctgcacactttttgctgctatcccaaaagctgtgtaaaataatcaaatg caaaataagcgcaagcagctgaaaacttctcttcaaacttttccgcttttcccaaaaccattttg ctttgaaatctgtttttaccaaatcagattaaactgcaaaatggaacttaaatgcaaatcattgc aatgcttataaactgttttt)

oskar region 2

(tgttctatatacttttgtgtgggtcaaaattcggcatgctcctgtatcacacaacctgccacttgccc ttaaaaagaagggcgcagtgggcgtggtacgtatacatatgagccatgctgcattttggccgt aatgaaaatgcactgctttacttggaaaattcgcttgcacaaaatcaacgccgcggctgattta ttattgatgtgctcaagcaaattcaagtgaagcatttgcgcgattttcgtctttctgtttccgtt)

oskar region 3

(tgcaaaaaagtttataaaatgcttacactctgctgcagacacgccaaccggaagtgcgcactaag cgcttgtttgtagcacagtgtagaattctggcgtaatttacagctctactttaaagtcttctagata gctatctactatttataaacttatttattgtcttgaatgtatgttaattgtatgtattgatggtgatca cgttttttttgtcctataacaagctgcaatgtaaaatccaaaaaaaaatgaaaaaataaaat aaaagggaaatcaaaaaaaaaaaaaaaaaaaaaaataaaaaaaaaaaaaaaggaatgc tgcggccgccaccgcggtggectaccaggggatcc)

oskar region $2 \mathrm{~b}$

(aatgaaaatgcactgctttacttggaaaattcgcttgcacaaaatcaacgccgcggctgattta ttattgatgtgctcaagcaaattcaagtgaagcatttgcgcgattttcgt)

oskar region $\Delta 2 \mathrm{~b}-\mathrm{p}$

(tgttctatatacttttgtgtgggtcaaaattcggcatgctcctgtatcacacaacctgccacttgccc ttaaaaagaagggcgcagtgggcgtggtacgtatacatatgagccatgctgcattttggccgt [...] tggaaaattcgcttgcacaaaatcaacgecgcggctgatttattattgatgtgctcaa gcaaattca $[. .$.$] ctttctgtttccgtttgcaaaaaagtt)$

oskar region $2 \Delta 2 \mathrm{~b}-\mathrm{d}$

(tgttctatatacttttgtgtgggtcaaaattcggcatgctcctgtatcacacaacctgccacttgccc ttaaaaagaagggcgcagtgggcgtggtacgtatacatatgagccatgctgcattttggccgt aatgaaaatgcactgctttact[...] agtgaagcatttgcgcgattttcgtctttctgtttccgtt tgcaaaaaagtt)

oskar region2 5'mut

(tgttctatatacttttgtgtgggtcaaaattcggcatgctcctgtatcacacaacctgccacttgccc ttaaaaagaagggcgcagtgggcgtggtacgtatacatatgagccatgctgcattttggccgt aatgaaaatgcactgctttacttggaaaattcgcttgcacaTTTAGTacgccgcggctga tttattattgatgtgctcaagcaaattcaagtgaagcatttgcgcgattttcgt)

oskar region 23 'mut

(tgttctatatacttttgtgtgggtcaaaattcggcatgctcctgtatcacacaacctgccacttgccc ttaaaaagaagggcgcagtgggcgtggtacgtatacatatgagccatgctgcattttggccgt aatgaaaatgcactgctttacttggaaaattcgcttgcacaaaatcaacgccgcggcACT AAAattattgatgtgctcaagcaaattcaagtgaagcatttgcgcgattttcgt)

oskar region $25^{\prime} 3^{\prime}$ mut

(tgttctatatacttttgtgtgggtcaaaattcggcatgctcctgtatcacacaacctgccacttgccc ttaaaaagaagggcgcagtgggcgtggtacgtatacatatgagccatgctgcattttggccgt aatgaaaatgcactgctttacttggaaaattcgcttgcacaTTTAGTacgccgcggc ACTAAAattattgatgtgctcaagcaaattcaagtgaagcatttgcgcgattttcgt)

oskar region $25 \mathrm{xGC}$

(tgttctatatacttttgtgtgggtcaaaattcggcatgctcctgtatcacacaacctgccacttgccc ttaaaaagaagggcgcagtgggcgtggtacgtatacatatgagccatgctgcattttggccgt aatgaaaatgcactgcccegtcctggaaattcgcttgcacaGGGCTGacgccgcggc CAGCCCattattgatgtgctcaagcaaattcaagtgaagcatttgcgcgattttcgtctt tctgtttccgtttgcaaaaaagtt)

oskar region $26 \mathrm{xGC}$

(tgttctatatacttttgtgtgggtcaaaattcggcatgctcctgtatcacacaacctgccacttgccc ttaaaaagaagggcgcagtgggcgtggtacgtatacatatgagccatgctgcattttggccgt aatgaaaatgcactgcccegtcctggaaattcgcttgcacaGGGCCGacgccgcggc CGGCCCattattgatgtgctcaagcaaattcaagtgaagcatttgcgcgattttcgtctt tctgtttccgtttgcaaaaaagtt)

oskar region2 $\Delta$ loop

(tgttctatatacttttgtgtgggtcaaaattcggcatgctcctgtatcacacaacctgccacttgccc ttaaaaagaagggcgcagtgggcgtggtacgtatacatatgagccatgctgcattttggccgt aatgaaaatgcactgctttacttggaaaattcgcttgcacaaaatcaacg[...]ctgatttatt attgatgtgctcaagcaaattcaagtgaagcatttgcgcgattttcgt)

\section{qRT-PCR}

Quantitative RT-PCR was performed on cDNAs generated from ovaries of flies expressing the respective transgenes. Amplified 
product was detected using the SYBRGreen (Ambion) system on an ABIPrism7500 real time PCR apparatus. The egfp portion of the transgenic RNAs was amplified using primers specific for the EGFP ORF and values normalized to $r p 49$. The amplification efficiency was determined using serial dilutions of a cDNA mix from all samples.

\section{Whole-mount in situ hybridization}

In situ hybridization was performed as described (Hachet and Ephrussi 2004). Antisense probes for the egfp and oskar coding sequences (cds) were generated by in vitro transcription (Ambion, Megascript) and detected using HRP-conjugated sheep anti-DIG antibody (1:200; Roche) followed by Cy3-tyramide signal amplification (Perkin Elmer). For all experiments at least 20 egg-chambers were scored to determine RNA localization.

\section{Fluorescent RNA synthesis and embryo injections}

Cy3-labeled RNAs were in vitro-transcribed and injected into blastoderm embryos as described (Bullock and Ish-Horowicz 2001). Embryos were fixed 8-10 min after injection (i.e., 8 min after injection of the last embryo). Scoring was performed using a Zeiss 510 confocal microscope. For each embryo, the extent of apical RNA localization was classified into one of four categories: ++ , efficient localization (the vast majority of fluorescent signal in the apical cytoplasm, where it concentrates in puncta apical to the nuclei); +, weak localization (substantial RNA signal in the basal cytoplasm, but clear concentration of RNA in apical puncta relative to the background signal); $+/-$, very weak localization (apical puncta only just visible above levels of fluorescence in basal cytoplasm); -, no apical localization (no discernible concentration of RNA in apical puncta). Scoring the same transcripts on different days of injection produced very similar results. The $f_{s}(1) K 10$ RNA corresponds to the entire 1432-nt $3^{\prime}$ UTR and an 860-nt portion of the $3^{\prime}$ genomic sequences. The TLS-mut RNA contains a randomized version of the TLS within the $f_{s}(1) K 10$ RNA (Bullock et al. 2010).

\section{Fly stocks}

$w^{1118}$ flies were used as the wild-type control, except for embryo injections, when Oregon-R flies were used. The oskar RNA null genotype was described in Jenny et al. (2006) and was trans-heterozygous for $0 s k^{\mathrm{A} 87}$ (Vanzo and Ephrussi 2002) and $D f(3 R) p^{X T 103}$ (Lehmann and Nusslein-Volhard 1986). UAS-coupled genes were expressed in the germline using $p$ Cog-Gal4:VP16 (Rorth et al. 1998), nanos-Gal4: VP16 (Rorth et al. 1998), and mat-a4-tub-Gal4:VP16 (Bloomington stock \#7062) promoters. Expression in follicle cells was under the control of GR1-Gal4 (Bloomington stock \#36287), and salivary gland expression was under the control of forkhead-Gal4 (Henderson and Andrew 2000).

\section{Microscopy}

Except for embryo injection assays (see above), a confocal microscope (DMR-E, Leica) equipped with a scan head (TCS SP2 AOBS; Leica) and an oil-immersion $20 \times 0.7$ NA objective (Leica) was used for image collection. Images were acquired at a $2 \times$ zoom and edited with Adobe Photoshop CS and FIJI (Schindelin et al. 2012). When necessary, images of egg-chambers were oriented along the anterior-posterior axis and images rendered rectangular.

\section{SUPPLEMENTAL MATERIAL}

Supplemental material is available for this article.

\section{ACKNOWLEDGMENTS}

We thank Pavel Tomancak, Imre Gaspar, and Simone Reber for their critical reading of the manuscript, W. Shakespeare for poetic inspiration regarding the term OES, and James Saenz and Sanjay Ghosh for discussions. S.L.B. was supported by UK MRC core funding (U105178790) and a Lister Institute Research Prize.

Received July 23, 2013; accepted January 6, 2014.

\section{REFERENCES}

Bastock R, St Johnston D. 2008. Drosophila oogenesis. Curr Biol 18: R1082-R1087.

Brendza RP, Serbus LR, Duffy JB, Saxton WM. 2000. A function for kinesin I in the posterior transport of oskar mRNA and Staufen protein. Science 289: 2120-2122.

Bullock SL, Ish-Horowicz D. 2001. Conserved signals and machinery for RNA transport in Drosophila oogenesis and embryogenesis. Nature 414: 611-616.

Bullock SL, Zicha D, Ish-Horowicz D. 2003. The Drosophila hairy RNA localization signal modulates the kinetics of cytoplasmic mRNA transport. EMBO J 22: 2484-2494.

Bullock SL, Ringel I, Ish-Horowicz D, Lukavsky PJ. 2010. A'-form RNA helices are required for cytoplasmic mRNA transport in Drosophila. Nat Struct Mol Biol 17: 703-709.

Cha BJ, Serbus LR, Koppetsch BS, Theurkauf WE. 2002. Kinesin I-dependent cortical exclusion restricts pole plasm to the oocyte posterior. Nat Cell Biol 4: 592-598.

Clark I, Giniger E, Ruohola-Baker H, Jan LY, Jan YN. 1994. Transient posterior localization of a kinesin fusion protein reflects anteroposterior polarity of the Drosophila oocyte. Curr Biol 4: 289-300.

Clark IE, Jan LY, Jan YN. 1997. Reciprocal localization of Nod and kinesin fusion proteins indicates microtubule polarity in the Drosophila oocyte, epithelium, neuron and muscle. Development 124: $461-470$.

Clark A, Meignin C, Davis I. 2007. A Dynein-dependent shortcut rapidly delivers axis determination transcripts into the Drosophila oocyte. Development 134: 1955-1965.

Cohen RS, Zhang S, Dollar GL. 2005. The positional, structural, and sequence requirements of the Drosophila TLS RNA localization element. RNA 11: 1017-1029.

Dienstbier M, Boehl F, Li X, Bullock SL. 2009. Egalitarian is a selective RNA-binding protein linking mRNA localization signals to the dynein motor. Genes Dev 23: 1546-1558.

dos Santos G, Simmonds AJ, Krause HM. 2008. A stem-loop structure in the wingless transcript defines a consensus motif for apical RNA transport. Development 135: 133-143.

Ephrussi A, Lehmann R. 1992. Induction of germ cell formation by oskar. Nature 358: 387-392.

Ephrussi A, Dickinson LK, Lehmann R. 1991. oskar organizes the germ plasm and directs localization of the posterior determinant nanos. Cell 66: 37-50.

Ghosh S, Marchand V, Gaspar I, Ephrussi A. 2012. Control of RNP motility and localization by a splicing-dependent structure in oskar mRNA. Nat Struct Mol Biol 19: 441-449. 
Glotzer JB, Saffrich R, Glotzer M, Ephrussi A. 1997. Cytoplasmic flows localize injected oskar RNA in Drosophila oocytes. Curr Biol 7: 326-337.

Hachet O, Ephrussi A. 2004. Splicing of oskar RNA in the nucleus is coupled to its cytoplasmic localization. Nature 428: 959-963.

Henderson KD, Andrew DJ. 2000. Regulation and function of Scr, exd, and hth in the Drosophila salivary gland. Dev Biol 217: 362-374.

Horne-Badovinac S, Bilder D. 2008. Dynein regulates epithelial polarity and the apical localization of stardust A mRNA. PLoS Genet 4: e8.

Jambor H, Brunel C, Ephrussi A. 2011. Dimerization of oskar 3' UTRs promotes hitchhiking for RNA localization in the Drosophila oocyte. RNA 17: 2049-2057.

Januschke J, Gervais L, Gillet L, Keryer G, Bornens M, Guichet A. 2006. The centrosome-nucleus complex and microtubule organization in the Drosophila oocyte. Development 133: 129-139.

Jenny A, Hachet O, Zavorszky P, Cyrklaff A, Weston MD, Johnston DS, Erdelyi M, Ephrussi A. 2006. A translation-independent role of oskar RNA in early Drosophila oogenesis. Development 133: 2827-2833.

Karlin-Mcginness M, Serano TL, Cohen RS. 1996. Comparative analysis of the kinetics and dynamics of K10, bicoid, and oskar mRNA localization in the Drosophila oocyte. Dev Genet 19: 238-248.

Kim-Ha J, Smith JL, Macdonald PM. 1991. oskar mRNA is localized to the posterior pole of the Drosophila oocyte. Cell 66: 23-35.

Kim-Ha J, Webster PJ, Smith JL, Macdonald PM. 1993. Multiple RNA regulatory elements mediate distinct steps in localization of oskar mRNA. Development 119: 169-178.

Kim-Ha J, Kerr K, Macdonald PM. 1995. Translational regulation of oskar mRNA by Bruno, an ovarian RNA-binding protein, is essential. Cell 81: 403-412.

King RC. 1970. Ovarian development in Drosophila melanogaster. Academic Press, New York.

Lecuyer E, Yoshida H, Parthasarathy N, Alm C, Babak T, Cerovina T, Hughes TR, Tomancak P, Krause HM. 2007. Global analysis of mRNA localization reveals a prominent role in organizing cellular architecture and function. Cell 131: 174-187.

Lehmann R, Nusslein-Volhard C. 1986. Abdominal segmentation, pole cell formation, and embryonic polarity require the localized activity of oskar, a maternal gene in Drosophila. Cell 47: 141-152.

Li Z, Wang L, Hays TS, Cai Y. 2008. Dynein-mediated apical localization of crumbs transcripts is required for Crumbs activity in epithelial polarity. J Cell Biol 180: 31-38.

Macdonald PM, Kerr K. 1998. Mutational analysis of an RNA recognition element that mediates localization of bicoid mRNA. Mol Cell Biol 18: 3788-3795.

Macdonald PM, Kerr K, Smith JL, Leask A. 1993. RNA regulatory element BLE1 directs the early steps of bicoid mRNA localization. Development 118: 1233-1243.

Mach JM, Lehmann R. 1997. An Egalitarian-BicaudalD complex is essential for oocyte specification and axis determination in Drosophila. Genes Dev 11: 423-435.

Markussen FH, Michon AM, Breitwieser W, Ephrussi A. 1995. Translational control of oskar generates Short OSK, the isoform that induces pole plasma assembly. Development 121: 3723-3732.

Martin KC, Ephrussi A. 2009. mRNA localization: Gene expression in the spatial dimension. Cell 136: 719-730.

McGrail M, Hays TS. 1997. The microtubule motor cytoplasmic dynein is required for spindle orientation during germline cell divisions and oocyte differentiation in Drosophila. Development 124: 24092419.

Medioni C, Mowry K, Besse F. 2012. Principles and roles of mRNA localization in animal development. Development 139: 3263-3276.

Myat MM, Andrew DJ. 2002. Epithelial tube morphology is determined by the polarized growth and delivery of apical membrane. Cell 111: 879-891.

Parton RM, Hamilton RS, Ball G, Yang L, Cullen CF, Lu W, Ohkura H, Davis I. 2011. A PAR-1-dependent orientation gradient of dynamic microtubules directs posterior cargo transport in the Drosophila oocyte. J Cell Biol 194: 121-135.
Pokrywka NJ, Stephenson EC. 1995. Microtubules are a general component of mRNA localization systems in Drosophila oocytes. Dev Biol 167: 363-370.

Rongo C, Gavis ER, Lehmann R. 1995. Localization of oskar RNA regulates oskar translation and requires Oskar protein. Development 121: 2737-2746.

Rorth P. 1998. Gal4 in the Drosophila female germline. Mech Dev 78: 113-118.

Rorth P, Szabo K, Bailey A, Laverty T, Rehm J, Rubin GM, Weigmann K, Milan M, Benes V, Ansorge W, et al. 1998. Systematic gain-of-function genetics in Drosophila. Development 125: 1049-1057.

Schindelin J, Arganda-Carreras I, Frise E, Kaynig V, Longair M, Pietzsch T, Preibisch S, Rueden C, Saalfeld S, Schmid B, et al. 2012. Fiji: An open-source platform for biological-image analysis. Nat Methods 9: 676-682.

Serano TL, Cohen RS. 1995. A small predicted stem-loop structure mediates oocyte localization of Drosophila K10 mRNA. Development 121: 3809-3818.

Singer RH. 1993. RNA zipcodes for cytoplasmic addresses. Curr Biol 3: 719-721.

Sinsimer KS, Jain RA, Chatterjee S, Gavis ER. 2011. A late phase of germ plasm accumulation during Drosophila oogenesis requires Lost and Rumpelstiltskin. Development 138: 3431-3440.

Smith JL, Wilson JE, Macdonald PM. 1992. Overexpression of oskar directs ectopic activation of nanos and presumptive pole cell formation in Drosophila embryos. Cell 70: 849-859.

Snee MJ, Arn EA, Bullock SL, Macdonald PM. 2005. Recognition of the $b c d$ mRNA localization signal in Drosophila embryos and ovaries. Mol Cell Biol 25: 1501-1510.

Snee MJ, Harrison D, Yan N, Macdonald PM. 2007. A late phase of Oskar accumulation is crucial for posterior patterning of the Drosophila embryo, and is blocked by ectopic expression of Bruno. Differentiation 75: 246-255.

Suter B, Steward R. 1991. Requirement for phosphorylation and localization of the Bicaudal-D protein in Drosophila oocyte differentiation. Cell 67: 917-926.

Swan A, Nguyen T, Suter B. 1999. Drosophila Lissencephaly-1 functions with Bic-D and dynein in oocyte determination and nuclear positioning. Nat Cell Biol 1: 444-449.

Theurkauf WE, Smiley S, Wong ML, Alberts BM. 1992. Reorganization of the cytoskeleton during Drosophila oogenesis: Implications for axis specification and intercellular transport. Development 115: 923936.

Theurkauf WE, Alberts BM, Jan YN, Jongens TA. 1993. A central role for microtubules in the differentiation of Drosophila oocytes. Development 118: 1169-1180.

Van De Bor V, Hartswood E, Jones C, Finnegan D, Davis I. 2005. gurken and the $I$ factor retrotransposon RNAs share common localization signals and machinery. Dev Cell 9: 51-62.

Van De Bor V, Zimniak G, Cerezo D, Schaub S, Noselli S. 2011. Asymmetric localisation of cytokine mRNA is essential for JAK/ STAT activation during cell invasiveness. Development 138: 13831393.

Vanzo NF, Ephrussi A. 2002. Oskar anchoring restricts pole plasm formation to the posterior of the Drosophila oocyte. Development 129: 3705-3714.

Vazquez-Pianzola P, Urlaub H, Suter B. 2011. Pabp binds to the osk $3^{\prime} \mathrm{UTR}$ and specifically contributes to osk mRNA stability and oocyte accumulation. Dev Biol 357: 404-418.

Wilkie GS, Davis I. 2001. Drosophila wingless and pair-rule transcripts localize apically by dynein-mediated transport of RNA particles. Cell 105: 209-219.

Zhao T, Graham OS, Raposo A, St Johnston D. 2012. Growing microtubules push the oocyte nucleus to polarize the Drosophila dorsalventral axis. Science 336: 999-1003.

Zimyanin VL, Belaya K, Pecreaux J, Gilchrist MJ, Clark A, Davis I, St Johnston D. 2008. In vivo imaging of oskar mRNA transport reveals the mechanism of posterior localization. Cell 134: 843-853. 

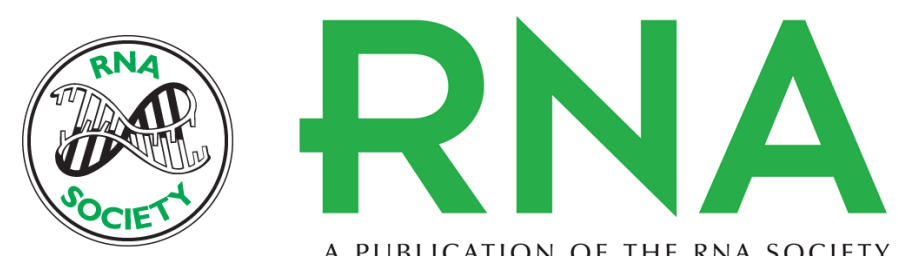

A PUBLICATION OF THE RNA SOCIETY

\section{A stem-loop structure directs oskar mRNA to microtubule minus ends}

Helena Jambor, Sandra Mueller, Simon L. Bullock, et al.

RNA 2014 20: 429-439 originally published online February 26, 2014

Access the most recent version at doi:10.1261/rna.041566.113

Supplemental Material

References

Open Access

Creative

Commons

License

Email Alerting
Service
http://rnajournal.cshlp.org/content/suppl/2014/02/11/rna.041566.113.DC1

This article cites 60 articles, 29 of which can be accessed free at: http://rnajournal.cshlp.org/content/20/4/429.full.html\#ref-list-1

Freely available online through the RNA Open Access option.

This article, published in $R N A$, is available under a Creative Commons License (Attribution-NonCommercial 3.0 Unported), as described at http://creativecommons.org/licenses/by-nc/3.0/.

Receive free email alerts when new articles cite this article - sign up in the box at the top right corner of the article or click here.

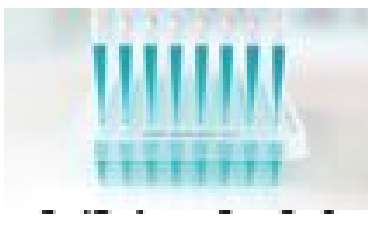

Providing Precise Solutions for your research.

To subscribe to $R N A$ go to:

http://rnajournal.cshlp.org/subscriptions

(C) 2014 Jambor et al.; Published by Cold Spring Harbor Laboratory Press for the RNA Society 